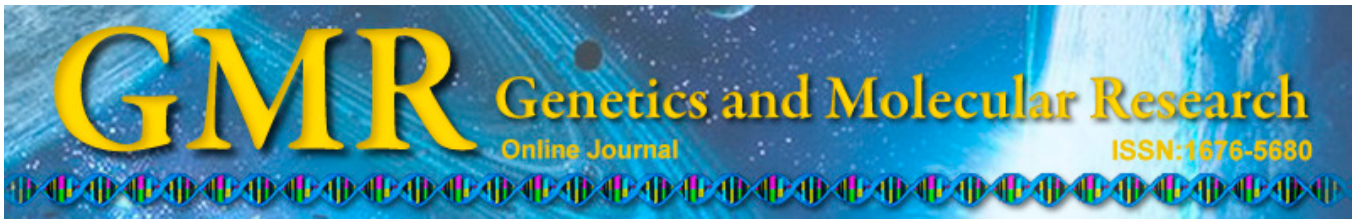

\title{
Systematic review of noninvasive prenatal diagnosis for abnormal chromosome genetic diseases using free fetal DNA in maternal plasma
}

\author{
H. Yang, H.B. Xu, T.T. Liu and X.L. He \\ Department of Obstetrics, \\ First Affiliated Hospital of Chongqing Medical University, Chongqing, China \\ Corresponding author: H.B. Xu \\ E-mail: xuhongbing_xhb@yeah.net
}

Genet. Mol. Res. 14 (3): 10603-10608 (2015)

Received January 24, 2015

Accepted April 29, 2015

Published September 9, 2015

DOI http://dx.doi.org/10.4238/2015.September.9.1

\begin{abstract}
We evaluated the system accuracy of noninvasive prenatal diagnosis for abnormal chromosome genetic diseases using cell-free fetal DNA in maternal plasma. Previous studies were searched in the MEDLINE database using the following keywords: "prenatal" and "aneuploidy" and "noninvasive or non-invasive" and "maternal". Identified studies were filtered using a QUADAS instrument. Four studies were identified and analyzed using QUADAS. The studies included 4167 cases of Down syndrome patients determined by noninvasive prenatal diagnosis with a sensitivity of $100 \%$ and specificity of $99.3 \%$; There were 3455 cases of Edwards syndrome patients determined by noninvasive prenatal diagnosis with a sensitivity of $97.4 \%$ and specificity of $99.95 \%$. Therefore, noninvasive prenatal diagnosis can be used to identify abnormal chromosomes with high accuracy using free fetal DNA in the maternal plasma.
\end{abstract}

Key words: Abnormal chromosome; Genetic diseases; Systematic review 


\section{INTRODUCTION}

Abnormal chromosomes can cause severe diseases resulting in fetal anomaly and hypoplasia and have an occurrence rate of 1 in 600-800 (Avent, 2013). Abnormal prenatal diagnosis began in the 1970s. Currently detection methods involve several steps. First, gravida is determined to identify if a woman is at high risk using serological and ultrasonographic methods, typically for Down's syndrome screening. Karyotyping or fluorescence in situ hybridization (FISH) (the traditional diagnosis method) is conducted to determine the presence of chromosome abnormalities in the fetus in the high-risk gravida (Qu et al., 2013). A limitation of this traditional diagnosis method is the false positive rate of $5 \%$ in Down's syndrome screening (Alldred, 2012), and karyotyping as well as FISH are invasive diagnosis methods that unnecessarily cause high-risk gravidas to suffer from the pain of chorionic villus, umbilical cord blood collection, and amniotic fluid puncture (Stumm et al., 2012).

Free fetal DNA in maternal plasma, reported by Lo et al. (1997), enables noninvasive diagnosis of fetal chromosome abnormalities. Sequencing and polymerase chain reaction (PCR) are conducted after extracting maternal blood to detect free fetal DNA in the maternal plasma (noninvasive diagnosis). Currently, fetal genetic diseases are determined relative to gender with free fetal DNA using the conventional clinical method (Liu et al., 2012). However, there is a large number of maternal free DNA segments in the maternal plasma, which is a challenge when identifying chromosome abnormalities (Simpson, 2013).

In this study, we analyzed relevant previous studies examining abnormal chromosome diagnosis with high accuracy using free fetal DNA in maternal plasma. Our results support the need for large-scale clinical studies and clinical applications of this method.

\section{MATERIAL AND METHODS}

\section{Search criteria}

The MEDLINE database was searched to identify studies published from 1997-2013 that included the following keywords: "prenatal" and "aneuploidy" and "noninvasive" or non-invasive and "maternal". The language was set to studies published in English or Chinese.

\section{Selected standard}

The following selection criteria were used: 1) the study objective was to determine the accuracy of noninvasive prenatal diagnosis for genetic diseases involving chromosome abnormalities by using free fetal DNA in the maternal plasma or serum; 2) the method for determining accuracy could be compared to the traditional standards, karyotyping and FISH.

\section{Literature selection}

Two groups of independent researchers experienced in the field evaluated the relatedness of the studies by examining the study titles and abstracts using the selection criteria. Studies meeting the selection criteria were included in our analysis, while studies showing inconsistencies were re-evaluated by experienced researchers by reading the full paper. When the same data had been published many times, only the latest publication was selected for our analysis. 


\section{Data collection}

All studies examined in this analysis were required to include accuracy analysis for noninvasive diagnosis and synchronized comparison analysis for the traditional karyotyping method. Sensitivity and specificity for all data for the noninvasive method were estimated for the studies selected by combination. The confidence interval was $95 \%$.

\section{RESULTS}

\section{Literature selection}

The selection method is shown in Figure 1. A total of 160 studies were identified using the keywords. By screening titles and abstracts, 22 studies related to noninvasive prenatal diagnosis were screened and selected for full-text reading. Among these, 15 studies were related to new technology and new methods, but did not examine noninvasive prenatal accuracy on a large-scale, and thus were excluded. Three of the 7 remaining studies were excluded because no standard control was used, no original data were included, or chromosomal abnormalities causing diseases were not examined. Four studies were included in this analysis.

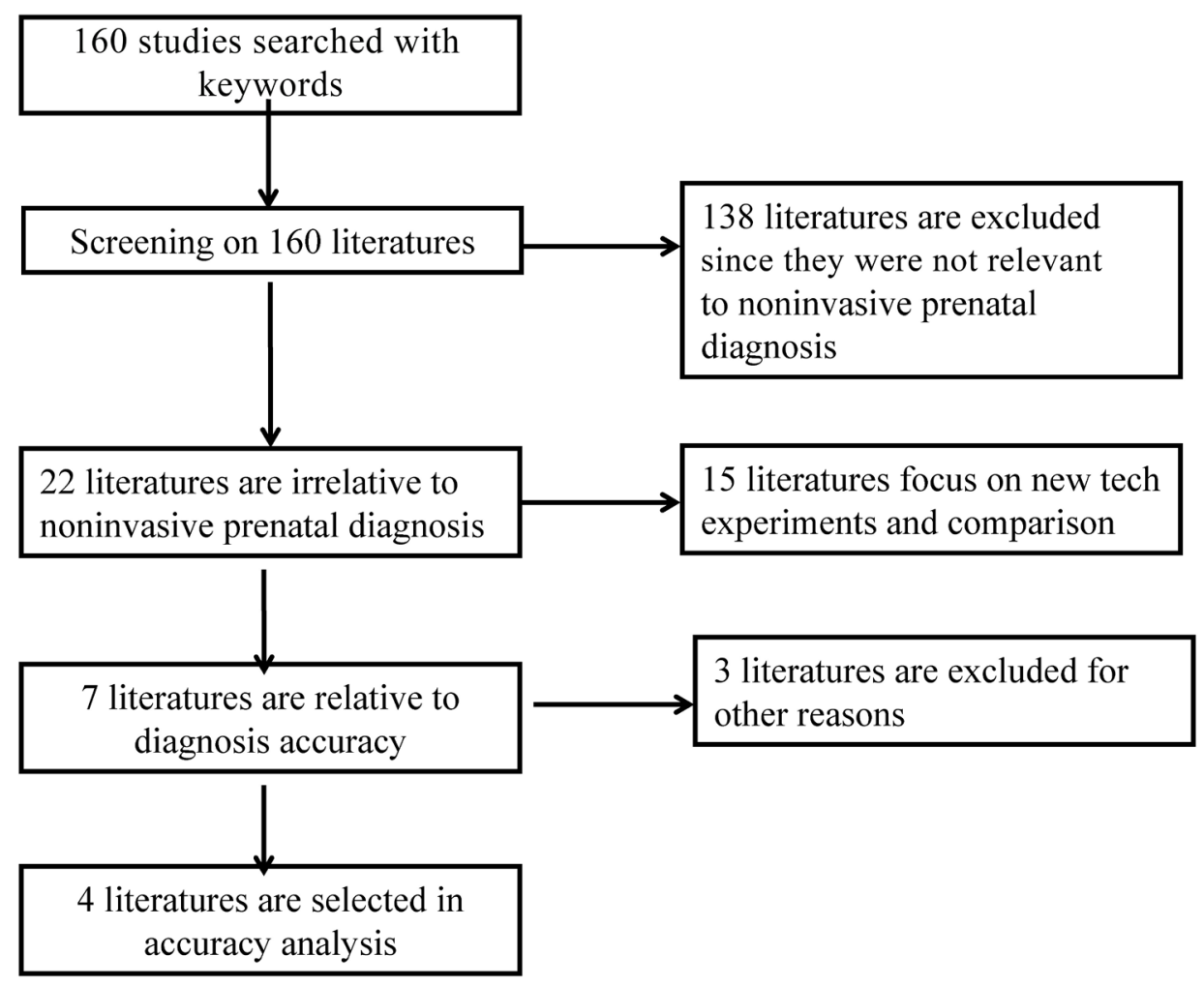

Figure 1. Study selection process. 


\section{Analysis}

The characteristics of the studies examined are shown in Tables 1 and 2. Four studies in total were selected for analysis. Lau et al. (2012) recently conducted noninvasive prenatal diagnosis of 567 high-risk gravida. Eight Down syndrome patients and 1 Edwards syndrome patient were diagnosed, which were found to be false-positive and false-negative by karyotyping. Noninvasive prenatal diagnosis has been shown to have an accuracy of $100 \%$ and specificity of $100 \%$, preventing gravidas from suffering through unnecessary invasive diagnosis with high false-positive rates.

\begin{tabular}{|c|c|c|c|c|c|c|c|}
\hline References & $\begin{array}{l}\text { Number of } \\
\text { gravidas }\end{array}$ & $\begin{array}{l}\text { Actual case number } \\
\text { of Down syndrome }\end{array}$ & $\begin{array}{c}\text { Case number } \\
\text { detected }\end{array}$ & $\begin{array}{l}\text { Case number of } \\
\text { false positive }\end{array}$ & Sensitivity & Specificity & Methods \\
\hline Lau et al. & 567 & 8 & 8 & 0 & $100 \%$ & $100.00 \%$ & Noninvasive sequencing \\
\hline Norton et al. & 2888 & 80 & 81 & 1 & $100 \%$ & $99.97 \%$ & Noninvasive sequencing \\
\hline Chiu et al. & 232 & 86 & 87 & 1 & $100 \%$ & $97.7 \%$ & Noninvasive sequencing \\
\hline Ehrich et al. & 480 & 39 & 40 & 1 & $100 \%$ & $99.7 \%$ & Noninvasive sequencing \\
\hline Sum & 4167 & 213 & 216 & 3 & $100 \%$ & $99.3 \%$ & \\
\hline
\end{tabular}

Table 2. Noninvasive prenatal diagnosis data for Edwards syndrome.
\begin{tabular}{lccccrcr}
\hline References & $\begin{array}{c}\text { Number of } \\
\text { gravidas }\end{array}$ & $\begin{array}{c}\text { Actual case number of } \\
\text { Edwards syndrome }\end{array}$ & $\begin{array}{c}\text { Case number } \\
\text { detected }\end{array}$ & $\begin{array}{c}\text { Case number of } \\
\text { false positive }\end{array}$ & Sensitivity & Specificity & Methods \\
\hline Lau et al. & 567 & 1 & 1 & 0 & $100.0 \%$ & $100.00 \%$ & Noninvasive sequencing \\
Norton et al. & 2888 & 38 & 37 & 2 & $97.4 \%$ & $99.93 \%$ & Noninvasive sequencing \\
Sum & 3455 & 39 & 38 & 2 & $97.4 \%$ & $99.95 \%$ & \\
\hline
\end{tabular}

Norton et al. (2012) reported that chromosomes 21 and 18 (used for determining highrisk) were analyzed with a sequencing method using the sample of free fetal DNA from the maternal plasma; the experimental design was a cohort study involving multiple centers. The results showed that 81 of the 2888 gravidas were Down syndrome patients, although 1 was false-positive. The detection rate was $100 \%$, while the false-positive rate was $0.03 \%$. In the 38 Edwards syndrome patients, 37 patients showed positive results, 2 of which were falsepositives. The detection rate was $97.4 \%$, while the false-positive rate was $0.07 \%$.

Chiu et al. (2011) analyzed 753 samples from gravidas and 314 gravidas separately using 8- and 2-plex sequencing. The detection rate of Down syndrome using 8-plex sequencing was $79.1 \%$ and the specificity was $98.0 \%$; the detection rate using 2-plex sequencing was $100 \%$ and the specificity was $97.7 \%$. The result was that 2 -plex method was selected in analysis due to 8-plex.

Ehrich et al. (2011) analyzed 480 samples of gravidas in 2011, and 13 samples could not be analyzed in our study because of insufficient sampling and quality issues. Eighteen samples of genome DNA passed quality control. There were a total of 449 samples, of which 39 cases were diagnosed as Down syndrome. One case was not Down syndrome with chromosome abnormality, showing a sensitivity of $100 \%$ and specificity of $99.95 \%$.

According to the literature data, the sensitivity of detection of Down syndrome using noninvasive sequence diagnosis was $100 \%$ and the specificity was $99.3 \%$; the sensitivity of detection of Edwards syndrome was $97.4 \%$ and the specificity was $99.95 \%$. 


\section{DISCUSSION}

Chromosome abnormalities can result in severe disease, causing fetal anomaly and hypoplasia with an occurrence rate of 1 in 600-800 (Avent, 2013). Prenatal diagnosis of abnormalities originated in 1970s. Currently, the gravida is first determined to be high-risk using serological and ultrasonographic methods for Down's syndrome screening. Karyotyping or FISH, steps in the traditional diagnosis method, is conducted to determine the presence of abnormal chromosomes in the fetus in high-risk gravidas (Qu et al., 2013). However, the traditional diagnosis method shows a false-positive rate of 5\% in Down's syndrome screening (Alldred et al., 2012), and karyotyping as well as FISH are invasive methods, causing highrisk gravidas to experience unnecessary pain for chorionic villus, umbilical cord blood collection, and amniotic fluid puncture.

Free fetal DNA in maternal plasma was reported by Lo et al. (1997), making noninvasive diagnosis for fetal chromosome abnormalities possible using noninvasive techniques such as PCR. Currently, conventional clinical methods are used to determine fetal genetic diseases resulting from chromosomal abnormalities based on gender, but the large number of maternal free DNA segments in the maternal plasma limit the ability to detect abnormal chromosomes.

Verweij et al. (2012) reported a systematic analysis of the accuracy of noninvasive sequencing for prenatal diagnosis of Down syndrome. Because of rapid developments in sequencing technology and improvements in free fetal DNA analysis technology, this technology has been expanded for diagnosing Edwards syndrome and other chromosome genetic diseases, as well as increased the sensitivity and specificity in diagnosis. However, sensitivity and specificity can change at different science research levels and experimental levels in various hospitals during clinical application. Additionally, since 2012, the ability to detect Down syndrome has greatly increased in specificity and sensitivity. Two recent studies showed sensitivities of $100 \%$ and specificities of 100 and $99.97 \%$. However, the sensitivity and specificity of Edwards syndrome diagnosis remain low. Thus, the development of methods for diagnosing individual chromosomal abnormalities requires further analysis.

Therefore, methods of noninvasive prenatal diagnosis of diseases involving chromosomal abnormalities using free fetal DNA in maternal plasma are sensitive and specific. The method used to diagnose Down syndrome has become more advanced, while the application for Edwards syndrome diagnosis requires additional research. The promotion of noninvasive sequencing prenatal diagnosis technology may be more widely used as the cost decreases.

\section{ACKNOWLEDGMENTS}

Research supported by a grant from the National Key Clinical Specialist.

\section{REFERENCES}

Alldred SK, Deeks JJ, Guo B, Neilson JP, et al. (2012). Second trimester serum tests for Down's Syndrome screening. Cochrane Database Syst. Rev. 6: CD009925.

Avent ND (2013). Maternal plasma biomarkers for down syndrome: present and future. Drugs Today 49: 145-152.

Chiu RW, Akolekar R, Zheng YW, Leung TY, et al. (2011). Non-invasive prenatal assessment of trisomy 21 by multiplexed maternal plasma DNA sequencing: large scale validity study. $B M J 342$ : $\mathrm{c} 7401$.

Ehrich M, Deciu C, Zwiefelhofer T, Tynan JA, et al. (2011). Noninvasive detection of fetal trisomy 21 by equencing of 
DNA in maternal blood: a study in a clinical setting. Am. J. Obstet. Gynecol. 204: 205.e1-205.e11.

Lau TK, Chan MK, Lo PS, Chan HY, et al. (2012). Clinical utility of noninvasive fetal trisomy (NIFTY) test - early experience. J. Matern. Fetal Neonatal Med. 25: 1856-1859.

Liu HY, Wu D, Li H, Guo SK, et al. (2012). Significance of detecting free DNA from maternal plasma for the diagnosis of fetal chromosomal aneuploidies. Zhonghua Yi Xue Yi Chuan Xue Za Zhi 29: 435-438.

Lo CM, Fan ST and Wong J (1997). The changing epidemiology of recurrent pyogenic cholangitis. Hong Kong Med. J. 3: 302-304.

Norton ME, Brar H, Weiss J, Karimi A, et al. (2012). Non-Invasive Chromosomal Evaluation (NICE) Study: results of a multicenter prospective cohort study for detection of fetal trisomy 21 and trisomy 18. Am. J. Obstet. Gynecol. 207: 137.e1-8.

Qu X, Randhawa G, Friedman C, O'Hara-Larrivee S, et al. (2013). A novel four-color fluorescence in situ hybridization assay for the detection of TMPRSS2 and ERG rearrangements in prostate cancer. Cancer Genet. 206: 1-11.

Simpson JL (2013). Cell-free fetal DNA and maternal serum analytes for monitoring embryonic and fetal status. Fertil. Steril. 99: 1124-1134.

Stumm M, Entezami M, Trunk N, Beck M, et al. (2012). Noninvasive prenatal detection of chromosomal aneuploidies using different next generation sequencing strategies and algorithms. Prenat. Diagn. 32: 569-577.

Verweij EJ, van den Oever JME, de Boer MA, Boon EM, et al. (2012). Diagnostic accuracy of noninvasive detection of fetal trisomy 21 in maternal blood: a systematic review. Fetal Siagnosis Ther. 31: 81-86. 